\title{
Atypical pathogens in hospitalized patients with community-acquired pneumonia: a worldwide perspective
}

\author{
Andrea Gramegna', Giovanni Sotgiu ${ }^{2}$, Marta Di Pasquale ${ }^{1}$, Dejan Radovanovic ${ }^{3}$, Silvia Terraneo ${ }^{4}$, Luis F. Reyes ${ }^{5}$, \\ Ester Vendrell ${ }^{6}$, Joao Neves ${ }^{7}$, Francesco Menzella ${ }^{8}$, Francesco Blasi ${ }^{1}$, Stefano Aliberti ${ }^{1 *}$, Marcos I. Restrepo ${ }^{5}$ \\ and on behalf of the GLIMP Study Group
}

\begin{abstract}
Background: Empirical antibiotic coverage for atypical pathogens in community-acquired pneumonia (CAP) has long been debated, mainly because of a lack of epidemiological data. We aimed to assess both testing for atypical pathogens and their prevalence in hospitalized patients with CAP worldwide, especially in relation with disease severity.

Methods: A secondary analysis of the GLIMP database, an international, multicentre, point-prevalence study of adult patients admitted for CAP in 222 hospitals across 6 continents in 2015, was performed. The study evaluated frequency of testing for atypical pathogens, including L. pneumophila, M. pneumoniae, C. pneumoniae, and their prevalence. Risk factors for testing and prevalence for atypical pathogens were assessed through univariate analysis.

Results: Among 3702 CAP patients 1250 (33.8\%) underwent at least one test for atypical pathogens. Testing varies greatly among countries and its frequency was higher in Europe than elsewhere (46.0\% vs. $12.7 \%$, respectively, $p<0$. 0001). Detection of L. pneumophila urinary antigen was the most common test performed worldwide (32.0\%). Patients with severe CAP were less likely to be tested for both atypical pathogens considered together (30.5\% vs. 35.0\%, $p=0.009$ ) and specifically for legionellosis ( $28.3 \%$ vs. $33.5 \%, p=0.003$ ) than the rest of the population. Similarly, L. pneumophila testing was lower in ICU patients. At least one atypical pathogen was isolated in 62 patients (4.7\%), including M. pneumoniae (26/251 patients, 10.3\%), L. pneumophila (30/1186 patients, 2.5\%), and C. pneumoniae (8/228 patients, 3.5\%). Patients with CAP due to atypical pathogens were significantly younger, showed less cardiovascular, renal, and metabolic comorbidities in comparison to adult patients hospitalized due to non-atypical pathogen CAP.

Conclusions: Testing for atypical pathogens in patients admitted for CAP in poorly standardized in real life and does not mirror atypical prevalence in different settings. Further evidence on the impact of atypical pathogens, expecially in the low-income countries, is needed to guidelines implementation.
\end{abstract}

Keywords: CAP, Atypical pathogens, Epidemiology

\footnotetext{
*Correspondence: stefano.aliberti@unimi.it

${ }^{1}$ Department of Pathophysiology and Transplantation, University of Milan,

Internal Medicine Department, Respiratory unit and Adult Cystic Fibrosis

Center, Fondazione IRCCS Ca' Granda Ospedale Maggiore Policlinico, Via

Francesco Sforza 35, 20122 Milan, Italy

Full list of author information is available at the end of the article
}

(c) The Author(s). 2018 Open Access This article is distributed under the terms of the Creative Commons Attribution 4.0 International License (http://creativecommons.org/licenses/by/4.0/), which permits unrestricted use, distribution, and reproduction in any medium, provided you give appropriate credit to the original author(s) and the source, provide a link to the Creative Commons license, and indicate if changes were made. The Creative Commons Public Domain Dedication waiver (http://creativecommons.org/publicdomain/zero/1.0/) applies to the data made available in this article, unless otherwise stated. 


\section{Background}

Community-acquired pneumonia (CAP) is a leading cause of hospitalization and death worldwide [1]. The annual estimated CAP burden in the Unites States of America (USA) accounts for more than 1.5 million adult hospitalizations and one third of hospitalized patients die within 1 year [2]. The assessment of the epidemiology of CAP-related pathogens is crucial to target appropriate empiric therapy in order to improve patients' outcomes. The empirical coverage for atypical pathogens, including Mycoplasma pneumoniae, Chlamydia pneumoniae, and Legionella pneumophila, is still a matter of debate [3].

Several Authors reported on an increased trend of atypical pathogens over the last 15 years, with prevalences ranging from 6 to $40 \%$ in both Europe and USA [4]. One study performed in China showed atypical pathogens as the most frequent cause of CAP with incidence rates far exceeding Streptococcus pneumoniae [5]. Other studies described similar prevalences of atypical pathogens [6].

Epidemiological data are mainly based on retrospective studies or secondary analyses of local or national datasets with key design limitations, such as: 1) cultures for atypicals are rarely performed and a standardized diagnostic approach has not been adopted; 2) serology for atypical pathogens could be prescribed for epidemiological studies according to international guidelines and an all-encompassing microbiological work-up should be carried out only for hospitalized patients with severe CAP $[1,7]$; 3) information on testing frequency of atypical pathogens and which population subgroups are more likely to be investigated are missing. Finally, the only published description on atypical pathogens in CAP is a secondary analysis of a retrospective database [6].

The aim of this study was to provide a real-life description of both testing frequency and prevalence of atypical pathogens in hospitalized patients with CAP worldwide, along with the evaluation of predisposing conditions for testing and risk factors for CAP caused by atypical pathogens.

\section{Methods}

\section{Study design and population}

The present study is based on a secondary analysis of the Global Initiative for MRSA Pneumonia (GLIMP) international database [8]. This project was not funded and relied upon voluntary site and investigator participation. The GLIMP methodology has been already published elsewhere [8]. The coordinating center (University of Texas Health at San Antonio -UT Health-, Texas, USA) received project approval by the Institutional Review Board (IRB\# HSC20150184E). All participating centers followed their local law and regulations. Study participants were enrolled on a single day in the months of March, April, May, and June in 2015.
All adults ( $>18$ years old) hospitalized with CAP were screened for study selection. CAP was defined by the evidence of new radiological pulmonary infiltrates during the first $48 \mathrm{~h}$ of hospitalization and by $\geq 1$ of the following criteria: 1) new or increased cough with/without sputum production and/or purulent respiratory secretions; 2) fever (documented rectal or oral temperature $\geq 37.8^{\circ} \mathrm{C}$ ) or hypothermia (documented rectal or oral temperature $<36^{\circ}$ C); 3) systemic inflammation (e.g., white blood cell count $>$ $10,000 / \mathrm{cm}^{3}$ or $<4000 / \mathrm{cm}^{3}$, C-reactive protein or procalcitonin values above the local upper limit of normality). Patients hospitalized with a diagnosis of hospital-acquired and/or ventilator-associated pneumonia were excluded. Patients without any bacterial tests for atypical pathogens collected within $24 \mathrm{~h}$ after hospital admission were also excluded.

Data collection and microbiology for atypical pathogens Data were collected using REDCap ${ }^{\mathrm{Tm}}$ (Research Electronic Data Capture), an electronic data capture tool hosted on the UT Health server. After study enrolment, participating centers were allowed 7 days to complete electronic data entry and confirm microbiological results.

Physicians taking care of CAP patients decided the microbiological work-up according to local standard operating procedures. Serology for atypical pathogens and urinary antigen test for L. pneumophila were performed by local hospital laboratories according to standard techniques. Atypical pathogens were considered: $M$. pneumoniae, $C$. pneumoniae, and L. pneumophila.

\section{Study groups}

Definition of CAP caused by atypical pathogens was based on species-specific serology or urinary antigen positivity. Patients tested for atypical pathogens were defined as having at least one of the following tests: urinary antigen test for L. pneumophila, serology for L. Pneumophila, C. pneumoniae, and M. pneumoniae.

\section{Study definitions}

CAP was deemed severe when patients were prescribed one of the following interventions: intensive care unit (ICU) admission, invasive or non-invasive mechanical ventilation, or vasopressor/inotrope administration during the first $24 \mathrm{~h}$ after hospital admission.

Definition of immunodepression was based on the diagnosis of $\geq 1$ of the following medical conditions in the six-month period before hospital admission: hematological malignancy, asplenia, aplastic anemia, neutropenia, long-term exposure to biological drugs or steroids or chemotherapy or immunosuppressive therapy for hematological/solid organ transplantation other than lung transplant, HIV/AIDS, and congenital or genetic immunodepression. All site investigators were 
provided with a protocol including the above-mentioned clinical definitions.

\section{Statistical analysis}

Testing frequency of atypical pathogens was calculated on all CAP patients in the dataset. Prevalence of an atypical pathogen was computed based on positive results of serology and/or urinary antigen test for L. pneumophila performed during the first $24 \mathrm{~h}$ of hospital stay. Categorical variables, expressed as absolute frequencies and percentages, were compared between groups using the Chi-squared test. Regressions analyses were performed to compare prevalence and compute odds ratios (OR) with their 95\% confidence interval (CI); furthermore, theywere performed to assess the relationship between atypical pathogen-related pneumonia and demographic, epidemiological, and clinical variables. Circular relation analysis using the Chi-squared test was performed to compare the prevalence between countries and continents. Statistical significance when $\mathrm{P}$ was $<0.05$. All statistical analyses were performed with IBM SPSS, Statistics for Mac, version 22.0, and STATA 13. Prevalence maps were created using Stat Planet software.

\section{Results}

\section{Testing for atypical pathogens}

A total of 3702 hospitalized CAP patients were recruited in 54 countries across 6 continents. Among them, 1250 (33.8\%) patients were tested for atypical pathogens: 1186 (32.0\%) for L. pneumophila (either urinary antigen or serology), 251 (6.8\%) for M. pneumoniae (serology), and 228 (6.1\%) for C. pneumoniae (serology). Distribution of testing frequencies across countries is showed in Fig. 1a.

The frequency of patients tested for atypicals was significantly higher in Europe in comparison with the rest of the world (46.0\% VS. $12.7 \%, P<0.0001)$. The lowest testing frequency was recorded in Africa and South America (5.8 and $5.0 \%$, respectively). The highest frequencies of patients tested for atypicals in countries enrolling $>100$ CAP patients were detected in Spain (70.8\%), Italy (63.8\%), Portugal (43.3\%), Germany (23.1\%), and USA (21.4\%) (Table 1). Data on testing for L. pneumophila, M. pneumoniae, and $C$. pneumoniae are reported in the additional files (Additional file 1: Table A). Detection of L. pneumophila urinary antigen was the most prevalent test performed worldwide (32.0\%).

The frequencies of patients tested for atypical pathogens were lower among those with severe CAP in comparison with those with non-severe CAP (30.5\% VS. 35.0\% for atypical pathogens other than L. pneumophila, $P=0.009 ; 28.3 \%$ VS. $33.5 \%$ for $L$. pneumophila, $P=0.003$ ). L. pneumophila testing was lower in ICU patients. Univariate analysis comparing characteristics of tested and non-tested patients is reported in Table 3, column A.

\section{Prevalence of atypical pathogens}

At least one atypical pathogen was isolated in 63 (4.7\%) patients out of those tested for atypicals. L. pneumophila was detected in 30 (2.5\%), M. pneumoniae in 26 (10.3\%), and C. pneumoniae in $8(3.5 \%)$ patients. The prevalence of atypical pathogens ranged from 0.0 to $36.4 \%$ and from 0.0 to $66.7 \%$ across different continents and countries, see Fig. 1b. Italy showed the highest prevalence of atypical pathogens in comparison with the rest of the world (7.5\% VS. $4.2 \%, P=0.022$ ), whereas Spain showed the lowest prevalence (2.2\% VS. $6.5 \%, P=0.001)$ (Table 2$)$.

Patients with CAP caused by atypical pathogens were significantly younger, showed less cardiovascular, renal, and metabolic comorbidities in comparison with patients with CAP caused by other pathogens CAP (Table 3, column B and Table 4).

\section{Discussion}

This secondary analysis of the GLIMP database found that only a third of patients hospitalized for CAP were tested for atypical pathogens worldwide, with a large variability among continents and countries. Patients with severe CAP were less likely to be tested for all atypical pathogens. Furthermore, L. pneumophila testing frequency was lower in ICU patients. Among those tested for, the prevalence of CAP caused by atypical pathogens was low. Younger age, female gender, and having a less comorbidities (cardiovascular disease, chronic renal failure) were factors associated with CAP due to atypicals.

The most frequent test for atypical pathogens performed in hospitalized patients with CAP was the Legionella urinary antigen (32.0\%), followed by Legionella serology, whereas frequency of serological testing for any atypical pathogens was very low (6.8 and $6.1 \%$ for $M$. pneumoniae and C. pneumoniae, respectively).

However, information on molecular biology was not retrieved in the GLIMP dataset based on missing recommendations by international guidelines [1, 7]. Although molecular techniques was found helpful in the diagnosis of CAP caused by L. pneumophila, findings from different studies showed that single available tests were not reliable for the detection of M. pneumoniae and C. pneumoniae in CAP patients [9-11]. In addition, molecular studies carried out in large population groups found financial limitations and lack of standardization $[6,12,13]$. Finally, these results are intended to be a real-life snapshot of what it is really done in different countries worldwide; we deem that it is unrealistic a worldwide shift to PCR techniques considering that data presented here suggest that even the most common and affordable test, the urinary antigen for Legionella, is not routinely prescribed.

One of the major implications of a poor standardized approach for atypical pathogen testing is the wide heterogeneity across continents and countries. In Europe, 


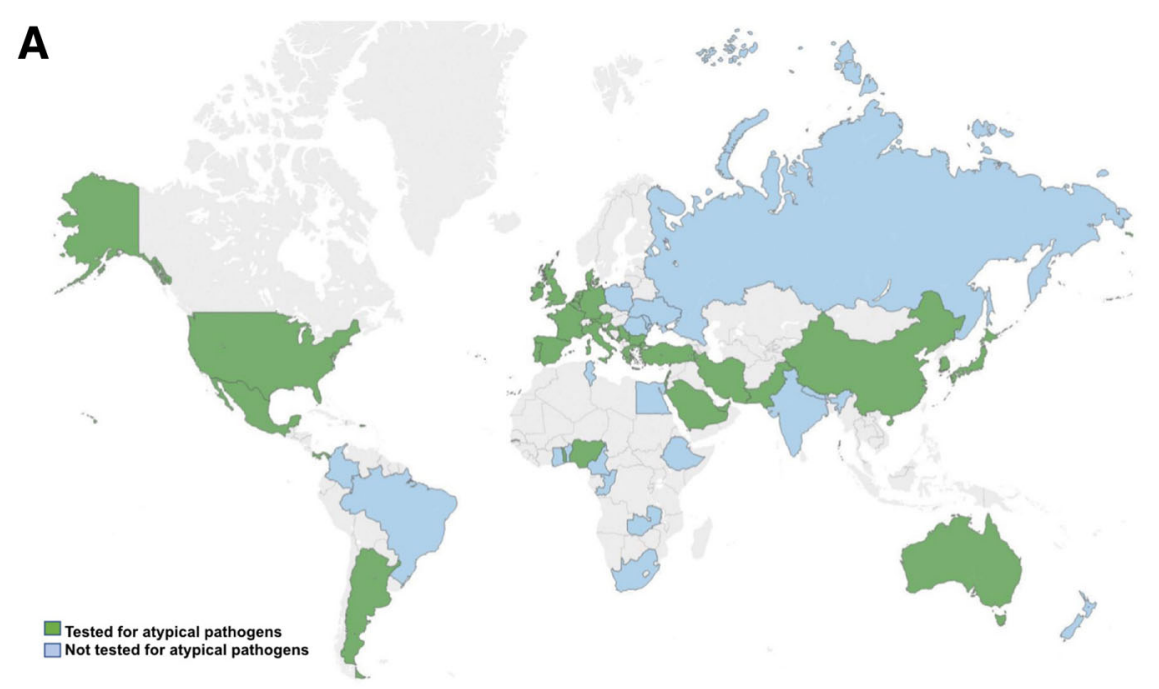

B

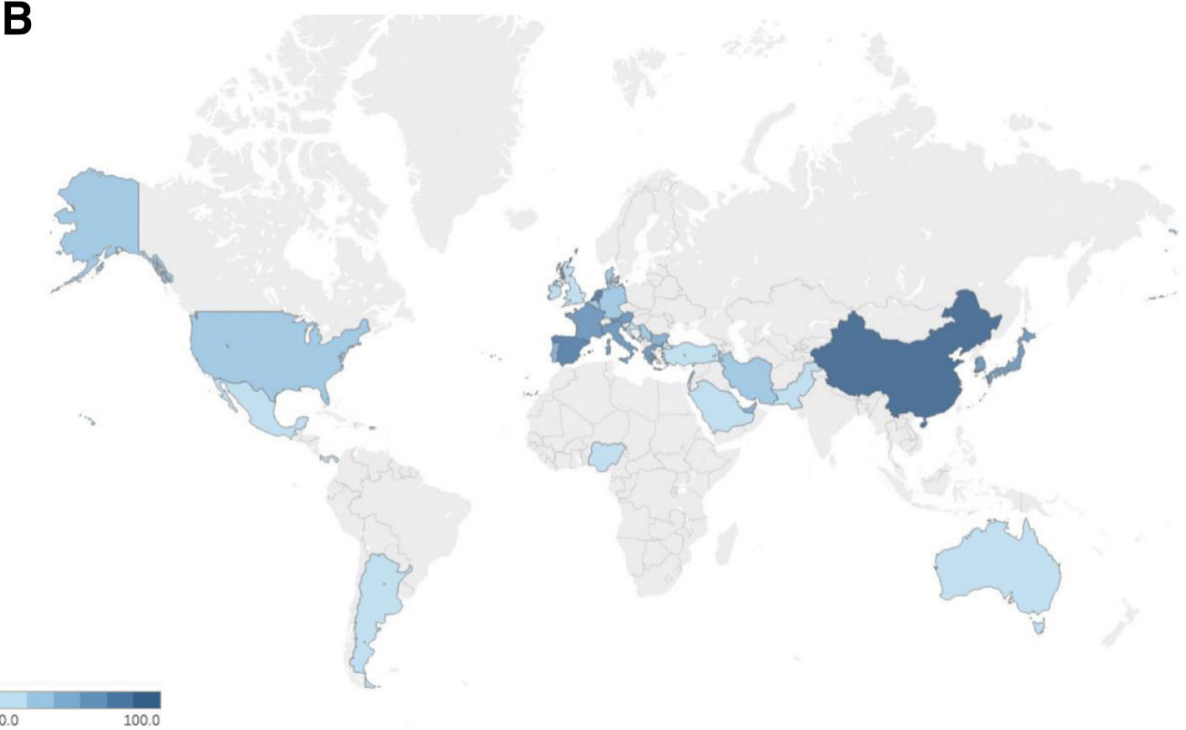

Fig. 1 a) Worldwide map on testing for atypical pathogens in hospitalized patients with community-acquired pneumonia (CAP) by country. Denominator: all CAP for each country. b) Worldwide map on prevalence of atypical pathogens in community-acquired pneumonia (CAP) in hospitalized patients by country. Denominator: all CAP tested for atypical pathogens for each country. Figure 1 is original and it was created using Stat Planet software with the information created from the study

almost half of the patients in the GLIMP database was investigated for atypical pathogens, thus resulting in the highest testing frequency. However, among European countries a significant variability was found. For example, the testing frequency was higher in the Mediterranean countries than in Northern Europe, ranging from $10.7 \%$ in United Kingdom to $70.8 \%$ in Spain. This significant difference may be caused by several factors, including the importance given to atypical pathogens in relation with national epidemiological reports and the lack of interest for this microbiological work-up in countries where extensive empirical therapy is routinely offered to patients. Interestingly, although large differences in frequencies of testing were found, prevalence of atypical pathogens seems to be quite similar in Europe, ranging from 1.6 to $6.5 \%$, with the only exception of Italy and Spain.

Furthermore, our data did not suggest significant clinical differences between patients who underwent testing for atypical pathogens and those who did not. The recent guidelines for the management of CAP published by the European Respiratory Society suggest a comprehensive microbiological work-up in severe patients [1]. However, we found that severe CAP was not a relevant driver for testing. Same results were obtained for other severity indicators, such as ICU admission, invasive/non-invasive mechanical ventilation, and administration of vasopressors. The low frequency testing may be explained by the 
Table 1 Testing frequency for atypical pathogens (all) in hospitalized patients with community-acquired pneumonia across different continents and countries

\begin{tabular}{|c|c|c|c|}
\hline Continent/Country & Tested/Total (\%) & Rest of the world Tested/Total (\%) & $P$ \\
\hline Europe & $1078 / 2344(46 \%)$ & $172 / 1358(12.7 \%)$ & $<0.0001$ \\
\hline North America & 105/529 (19.8\%) & $1145 / 3173(36.1 \%)$ & $<0.0001$ \\
\hline Asia & $44 / 415(10.6 \%)$ & 1206/3287 (36.7\%) & $<0.0001$ \\
\hline Oceania & $3 / 40(7.5 \%)$ & $1247 / 3662(34.1 \%)$ & $<0.0001$ \\
\hline Africa & 9/156 (5.8\%) & $1241 / 3546(35 \%)$ & $<0.0001$ \\
\hline South America & $11 / 218(5 \%)$ & 1239/3484 (35.6\%) & $<0.0001$ \\
\hline Spain & 455/643 (70.8\%) & $795 / 3059$ (26\%) & $<0.0001$ \\
\hline Italy & 293/459 (63.8\%) & $957 / 3243(29.5 \%)$ & $<0.0001$ \\
\hline Greece & $54 / 87(62.1 \%)$ & 1196/3615 (33.1\%) & $<0.0001$ \\
\hline France & $40 / 66(60.6 \%)$ & $1210 / 3636(33.3 \%)$ & $<0.0001$ \\
\hline Portugal & $58 / 134(43.3 \%)$ & $1192 / 3568(33.4 \%)$ & 0.018 \\
\hline Bulgaria & $21 / 51(41.2 \%)$ & 1229/3651 (33.7\%) & 0.260 \\
\hline Denmark & $26 / 89(29.2 \%)$ & 1224/3613 (33.9\%) & 0.358 \\
\hline Germany & 40/173 (23.1\%) & $1210 / 3529(34.3 \%)$ & 0.002 \\
\hline US & 102/477 (21.4\%) & $1148 / 3225$ (35.6\%) & $<0.0001$ \\
\hline Serbia & 10/56 (17.9\%) & 1240/3646 (34\%) & 0.011 \\
\hline Croatia & 16/103 (15.5\%) & 1234/3599 (34.3\%) & $<0.0001$ \\
\hline UK & 20/186 (10.8\%) & 1230/3516 (35\%) & $<0.0001$ \\
\hline Argentina & 11/190 (5.8\%) & $1239 / 3512(35.3 \%)$ & $<0.0001$ \\
\hline Pakistan & 6/109 (5.5\%) & $1244 / 3593$ (34.6\%) & $<0.0001$ \\
\hline
\end{tabular}

recommendation of several guidelines on broad empirical coverage in severe patients [1, 14-16]. Notably, despite cost-effectiveness and ease of use of urinary antigen test, L. pneumophila testing frequency was also lower in ICU patients. These data are consistent with those showed by Singanayagam who demonstrated that pneumonia severity scores, such as PSI and CURB-65, are poor predictors of microbial etiology and that atypical pathogens are more prevalent in patients with less disease severity at their presentation [17].

The present study showed that the estimated prevalence of atypical pathogens in hospitalized CAP patients is low during a non-epidemic season (i.e., from March to June).

Table 2 Prevalence of atypical pathogens in hospitalized patients with community-acquired pneumonia across different continents

\begin{tabular}{llll}
\hline Continent & Tested/Total (\%) & $\begin{array}{l}\text { Rest of the world } \\
\text { Tested/Total (\%) }\end{array}$ & $P$ \\
\hline Africa & $2 / 9(22.2 \%)$ & $60 / 1241(4.8 \%)$ & 0.070 \\
Asia & $2 / 44(4.5 \%)$ & $60 / 1206(5 \%)$ & 1 \\
Europe & $50 / 1078(4.6 \%)$ & $12 / 172(7 \%)$ & 0.091 \\
North America & $6 / 105(5.7 \%)$ & $56 / 1145(4.9 \%)$ & 0.922 \\
Oceania & $0 / 3(0 \%)$ & $62 / 1247(5 \%)$ & 1 \\
South America & $5 / 11(45 \%)$ & $57 / 1239(4.6 \%)$ & 0.001 \\
\hline
\end{tabular}

The proportional distribution was heterogeneous and the majority of the reported cases were from Europe. Intercontinent differences suggest that prevalence is lower in Africa and South America. L. pneumophila and M. pneumoniae seem to be the most frequent pathogens worldwide. The prevalence of M. pneumoniae is highest in South America, whereas L. pneumophila did show a homogeneous geographical distribution. L. pneumophila prevalence was similar to that recorded by Viasus (5.4\% among 3934 immunocompetent hospitalized CAP patients after a 15-year study) [18]. Conversely, our data might underestimate the high incidence of legionellosis (12\%) in the US population as previously reported by Vergis [19].

The CAPO database reported on a prevalence for atypical pathogens ranging from 20 to $28 \%$ across 21 countries over a five-year period (epidemic seasons included) [6]. The Authors performed a very comprehensive microbiological work-up including PCR for atypicals for the majority of CAP patients, but it is unclear the proportion of cases diagnosed by serological or molecular techniques. National and regional epidemiological reports showed a prevalence ranging from 9 to $50 \%$ [20-24]. Singanayagam and Coworkers recently published a secondary analysis of four independent prospective CAP datasets with atypical pathogens accounting for a global frequency of $14 \%$ in patients with identified microbiological positivity [17]. Interestingly, most of these 
Table 3 Clinical characteristics of tested and non-tested patients for both all atypical pathogens and L. pneumophila (column A) and of patients with community-acquired pneumonia caused and not caused by atypical pathogen (column B)

\begin{tabular}{|c|c|c|c|c|c|c|}
\hline \multirow[t]{2}{*}{ Variables } & \multicolumn{3}{|l|}{ Column A } & \multicolumn{3}{|l|}{ Column B } \\
\hline & $\begin{array}{l}\text { Tested patients } \\
(N=1250)\end{array}$ & $\begin{array}{l}\text { Non-tested patients } \\
(N=2452)\end{array}$ & P & $\begin{array}{l}\text { Atypical pathogen } \\
\text { CAP }(N=63)\end{array}$ & $\begin{array}{l}\text { Non-atypical pathogen } \\
\text { CAP }(N=1187)\end{array}$ & $P$ \\
\hline \multicolumn{7}{|l|}{ Demographic characteristics } \\
\hline Age, years & $68(46-75)$ & $70(51-81)$ & 0.43 & $62(43-72)$ & $71(56-81)$ & 0.015 \\
\hline Male, $n(\%)$ & $714(57)$ & 1459 (59) & 0.83 & $27(43)$ & $687(58)$ & 0.027 \\
\hline Underweight, n (\%) & $56(4.5)$ & $110(4.5)$ & 0.36 & $3(4.8)$ & $53(4.5)$ & 1 \\
\hline Obesity, $n(\%)$ & $208(16.6)$ & $369(15)$ & 0.21 & $11(17.4)$ & $197(16.6)$ & 0.811 \\
\hline \multicolumn{7}{|l|}{ Respiratory past medical history } \\
\hline Active lung cancer, s (\%) & $27(2.2)$ & $82(3.3)$ & 0.50 & $0(0)$ & $27(2.3)$ & 0.64 \\
\hline Asthma, $n(\%)$ & $85(6.8)$ & $176(7.2)$ & 0.73 & $3(4.8)$ & $82(6.9)$ & 0.79 \\
\hline Bronchiectasis, $n$ (\%) & $61(4.9)$ & $117(4.6)$ & 0.87 & $1(1.6)$ & $60(5)$ & 0.36 \\
\hline Chronic aspiration, $n$ (\%) & $71(5.7)$ & $186(7.6)$ & 0.03 & $0(0)$ & $71(6)$ & 0.45 \\
\hline COPD, n (\%) & $327(26.2)$ & $609(24.8)$ & 0.38 & $14(22.2)$ & $313(26.3)$ & 0.51 \\
\hline FEV1 $\leq 30 \%, n(\%)$ & $27(2.2)$ & $73(3)$ & 0.16 & $0(0)$ & $27(2.2)$ & 0.64 \\
\hline Current/former smoker, $n$ (\%) & $427(34.2)$ & $818(33.4)$ & 0.63 & $18(28.5)$ & $409(34.4)$ & 0.382 \\
\hline Interstitial lung disease, $n$ (\%) & $34(2.7)$ & $61(2.5)$ & 0.66 & $1(1.6)$ & $33(2.8)$ & 1 \\
\hline Obstructive sleep apnea, $n$ (\%) & $51(4.1)$ & $79(3.2)$ & 0.19 & $0(0)$ & $51(4.3)$ & 0.17 \\
\hline Oxygen therapy at home, $n(\%)$ & $83(6.6)$ & $141(5.7)$ & 0.30 & $4(6.4)$ & $79(6.6)$ & 1 \\
\hline Lung transplantation, $n(\%)$ & $1(0.8)$ & $6(0.2)$ & 0.44 & $0(0)$ & $1(0.8)$ & 1 \\
\hline Tracheostomy, n (\%) & $15(1)$ & $38(1.5)$ & 0.45 & $0(0)$ & $15(1.3)$ & 1 \\
\hline \multicolumn{7}{|l|}{ Cardiovascular past medical history } \\
\hline Arrhythmia, $n(\%)$ & $218(17.4)$ & $309(12.6)$ & $<0.001$ & $11(17.7)$ & $207(17.4)$ & 0.947 \\
\hline Coronary artery disease, $n(\%)$ & $178(14.2)$ & $345(14.1)$ & 0.88 & $3(4.8)$ & $175(14.7)$ & 0.030 \\
\hline Heart failure, $n(\%)$ & $88(7)$ & $210(8.2)$ & 0.26 & $2(3.2)$ & $86(7.2)$ & 0.312 \\
\hline Hypertension, $n$ (\%) & $183(14.6)$ & $323(13)$ & 0.14 & $4(6.5)$ & $179(14.4)$ & 0.790 \\
\hline \multicolumn{7}{|l|}{ Chronic medications } \\
\hline Inhaled corticosteroids use, $n(\%)$ & $207(16.6)$ & $383(15.6)$ & 0.47 & $4(6.5)$ & $203(17.1)$ & 0.028 \\
\hline Proton Pump Inhibitor use, $n(\%)$ & $401(32)$ & $627(25.6)$ & $<0.001$ & $17(27.4)$ & $384(32.3)$ & 0.423 \\
\hline Statins use, $n(\%)$ & $285(22.8)$ & $470(19.2)$ & 0.011 & $9(14.5)$ & $276(23.2)$ & 0.111 \\
\hline Steroids use, $n(\%)$ & $86(6.8)$ & $208(8.5)$ & 0.09 & $4(6.5)$ & $82(6.9)$ & 1 \\
\hline \multicolumn{7}{|l|}{ Chronic interventions } \\
\hline Enteric tube feeding, $n(\%)$ & $11(0.88)$ & $41(1.7)$ & 0.05 & $0(0)$ & $11(1)$ & 1 \\
\hline Haemodialysis, $n$ (\%) & $12(1)$ & $40(1.6)$ & 0.11 & $0(0)$ & $12(1)$ & 1 \\
\hline Indwelling catheter, $n$ (\%) & $18(1.4)$ & $61(2.5)$ & 0.04 & $2(3.2)$ & $16(1.4)$ & 0.22 \\
\hline \multicolumn{7}{|l|}{ Immunosuppressive conditions } \\
\hline Active solid tumour, $n(\%)$ & $88(7)$ & $199(8.1)$ & 0.27 & $1(1.6)$ & $87(7.3)$ & 0.12 \\
\hline HIV infection, $n(\%)$ & $28(2.24)$ & $95(3.9)$ & 0.009 & $2(3.2)$ & $26(2.2)$ & 0.64 \\
\hline AIDS, $n(\%)$ & $15(1.2)$ & $50(2)$ & 0.08 & $2(3.2)$ & $13(1.1)$ & 0.16 \\
\hline Aplastic anaemia, $n(\%)$ & $6(0.3)$ & $8(0.3)$ & 0.57 & $0(0)$ & $6(0.5)$ & 1 \\
\hline Asplenia, $n(\%)$ & $6(0.3)$ & $6(0.2)$ & 0.24 & $0(0)$ & $6(0.5)$ & 1 \\
\hline Biological drug use, $n(\%)$ & $14(1.1)$ & $23(0.9)$ & 0.60 & $0(0)$ & $14(1.2)$ & 1 \\
\hline Chemotherapy in the last 3 months, $n(\%)$ & $48(3.8)$ & $97(3.8)$ & 0.92 & $1(1.6)$ & $47(4)$ & 0.51 \\
\hline Haematological malignancy, $n$ (\%) & $73(5.8)$ & $89(3.6)$ & $=0.003$ & $2(3.2)$ & $71(6)$ & 0.57 \\
\hline Immunocompromised patients, $n(\%)$ & $230(18.4)$ & $435(17.7)$ & 0.62 & $12(19.4)$ & $218(18.4)$ & 0.84 \\
\hline
\end{tabular}


Table 3 Clinical characteristics of tested and non-tested patients for both all atypical pathogens and L. pneumophila (column A) and of patients with community-acquired pneumonia caused and not caused by atypical pathogen (column B) (Continued)

\begin{tabular}{|c|c|c|c|c|c|c|}
\hline \multirow[t]{2}{*}{ Variables } & \multicolumn{3}{|l|}{ Column A } & \multicolumn{3}{|l|}{ Column B } \\
\hline & $\begin{array}{l}\text { Tested patients } \\
(N=1250)\end{array}$ & $\begin{array}{l}\text { Non-tested patients } \\
(N=2452)\end{array}$ & P & $\begin{array}{l}\text { Atypical pathogen } \\
\text { CAP }(N=63)\end{array}$ & $\begin{array}{l}\text { Non-atypical pathogen } \\
\text { CAP }(N=1187)\end{array}$ & $P$ \\
\hline Neutropenia, n (\%) & $13(1.8)$ & $35(1.4)$ & 0.36 & $0(0)$ & $13(1.1)$ & 1 \\
\hline \multicolumn{7}{|l|}{ Other chronic medical conditions } \\
\hline Chronic renal failure, $n(\%)$ & $144(11.5)$ & $256(10.4)$ & 0.31 & $2(3.2)$ & $142(12)$ & 0.036 \\
\hline Dementia, $n(\%)$ & $136(18.9)$ & $272(11.1)$ & 0.87 & $5(8.1)$ & $131(11)$ & 0.46 \\
\hline Diabetes mellitus, $n(\%)$ & $266(21.3)$ & $516(21)$ & 0.86 & $7(11.3)$ & $259(21.8)$ & 0.049 \\
\hline Liver disease, $n(\%)$ & $59(4.72)$ & $81(3.03)$ & 0.36 & $4(6.5)$ & $55(4.6)$ & 0.53 \\
\hline Malnutrition, $n(\%)$ & $95(7.6)$ & $0(0)$ & 0.08 & $4(6.5)$ & $91(7.7)$ & 1 \\
\hline Mental illness, $n(\%)$ & $83(6.6)$ & $0(0)$ & 0.73 & $4(6.5)$ & $79(6.6)$ & 1 \\
\hline Prosthetic material, $n$ (\%) & $41(3.3)$ & $75(3)$ & 0.76 & $1(1.6)$ & $40(3.4)$ & 0.71 \\
\hline Recurrent skin infections, $n$ (\%) & $14(1.1)$ & $44(1.8)$ & 0.13 & $1(1.6)$ & $13(1.1)$ & 0.51 \\
\hline \multicolumn{7}{|l|}{ Other non-medical conditions } \\
\hline Bedridden, $n(\%)$ & $110(8.8)$ & $305(12.4)$ & 0.001 & $3(4.8)$ & $86(7.2)$ & 0.61 \\
\hline Contact sport, $n(\%)$ & $1(0.1)$ & 5 & 0.67 & $0(0)$ & $1(1)$ & 1.0 \\
\hline Healthcare worker, $n$ (\%) & $20(1.6)$ & $27(1.1)$ & 0.21 & $5(7.9)$ & $15(1.3)$ & 0.002 \\
\hline Homeless, $n(\%)$ & $12(1.8)$ & $23(0.9)$ & 1.0 & $0(0)$ & $12(1)$ & 1 \\
\hline Living in crowded conditions, $n$ (\%) & $236(18.9)$ & $485(19.8)$ & 0.54 & $0(0)$ & $9(0.8)$ & 1 \\
\hline Nursing home resident, $n$ (\%) & $86(6.88)$ & $216(8.8)$ & 0.042 & $11(17.7)$ & $225(18.9)$ & 0.81 \\
\hline Chronic aspiration, $n$ (\%) & $71(5.7)$ & $186(7.6)$ & 0.034 & $0(0)$ & $62(5.3)$ & 0.047 \\
\hline \multicolumn{7}{|l|}{ Previous infections/colonization } \\
\hline Prior mycobacterial diseases, $n(\%)$ & $31(2.5)$ & $65(2.6)$ & 0.82 & $3(4.8)$ & $28(2.4)$ & 0.19 \\
\hline Prior MRSA infection/colonisation, $n$ (\%) & $30(2.4)$ & $56(2.3)$ & 0.82 & $0(0)$ & $30(2.5)$ & 0.39 \\
\hline Prior ESBL-producing bacterial infection, $n(\%)$ & $21(1.7)$ & $34(1.4)$ & 0.48 & $1(1.6)$ & $20(1.7)$ & 1 \\
\hline Prior Pseudomonas spp. infection, $n(\%)$ & $30(2.4)$ & $71(2.9)$ & 0.45 & $1(1.6)$ & $29(2.4)$ & 1 \\
\hline \multicolumn{7}{|l|}{ Current pneumonia episode } \\
\hline Severe CAP, $n(\%)$ & $314(25.1)$ & $716(29.2)$ & 0.009 & $21(33)$ & $293(24.7)$ & 0.103 \\
\hline ICU or HDU admission, $n(\%)$ & $277(22.2)$ & $619(25.2)$ & $=0.039$ & $18(28)$ & $259(22)$ & 0.181 \\
\hline Either invasive or non-invasive ventilation, $n(\%)$ & $206(16.5)$ & $456(17.9)$ & 0.11 & $12(19)$ & $194(16.3)$ & 0.531 \\
\hline Invasive ventilation, $n$ (\%) & $114(9.1)$ & $240(9.4)$ & 0.55 & $3(4.8)$ & $111(9.3)$ & 0.230 \\
\hline Non-invasive ventilation, $n(\%)$ & $118(9.4)$ & $231(9)$ & 1 & $9(14.3)$ & $109(9.1)$ & 0.161 \\
\hline
\end{tabular}

CAP; Community-acquired pneumonia, MRSA; Methicillin resistant Staphylococcus aureus, COPD; Chronic obstructive pulmonary disease, FEV ${ }_{1}$; Forced expiratory volume during the first second, CAD; Coronary artery disease, ESBL; extended-spectrum beta-lactamases, LRTI; lower respiratory tract infections

studies suggested that atypical pathogens are more relevant in the outpatient population [17, 20-24].

The prevalence estimates on atypical microorganisms might be limited. Even if the combination of serology and molecular techniques was suggested to increase sensitivity, diagnostic tools only accounted on serology for atypical pathogens and urinary antigen for Legionella $[1,25]$. Then, prevalence estimation can depend on frequency and comprehensiveness of the microbiological work-up.

Second, since patients have been enrolled on a single day in the months of March, April and May, most of data come from non-epidemic season in northern hemisphere, thus biasing a plausible estimation of atypical pathogen epidemiology.

However, the low testing frequency underscores the poor emphasis given by physicians or local health authorities to the role of atypicals. Therefore, the controversy on empiric coverage for atypical pathogens should be addressed after a more adequate description of the epidemiological burden and a sensitization of attending physicians.

Potential risk factors for atypical pathogens were also investigated. In this analysis cardiovascular disease as well as chronic renal failure act as protective factors for atypical etiology. Our understanding is that these results 
Table 4 Protective factors for atypical pathogens in hospitalized patients with community-acquired pneumonia

\begin{tabular}{lll}
\hline & OR $(95 \% \mathrm{Cl})$ & $P$ \\
\hline Age & $0.583(0.350-0.973)$ & 0.039 \\
Cardiovascular disease & $>0.0001$ & $<0.0001$ \\
Diabetes mellitus & $0.464(0.207-1.043)$ & 0.063 \\
Chronic renal failure & $0.203(0.480-0.865)$ & 0.031 \\
Severe CAP & $1.769(0.516-3.073)$ & 0.364 \\
Mechanical ventilation & $0.288(0.810-1.031)$ & 0.056 \\
ICU admission & $1.156(0.311-4.294)$ & 0.826 \\
\hline
\end{tabular}

ICU: intensive care unit; OR: Odds ratio; Cl: confidence interval

might be a function of age, being patients with atypical pneumonia younger than others.

\section{Conclusions}

In conclusion, this real-life study demonstrates that testing for atypical pathogens in hospitalized patients with CAP is not routinely performed worldwide.

Testing for atypical pathogens is poorly standardized and a wide inter-country heterogeneity was found. Testing rates could not appropriately describe prevalence of atypicals in different settings. Further studies are needed to better assess the epidemiological burden and the utility of the current microbiological and clinical recommendations.

\section{Additional file}

Additional file 1: Table A: Tables with testing frequencies for specific atypical pathogens across continents (A1: Testing frequencies for $C$. pneumoniae across continents; A2: Testing frequencies for $M$. pneumoniae across continents; A3: Testing frequencies for L. pneumophila across continents).- Brief description of the data: a table in three parts reporting data about frequency of testing for different atypical pathogens across different continents. (DOC $50 \mathrm{~kb}$ )

\section{Abbreviations}

CAP: Community-acquired pneumonia; GLIMP: Global Initiative for MRSA Pneumonia; ICU: Intensive care unit

\footnotetext{
Acknowledgments

GLIMP investigators

Argentina-Patricia Karina Aruj (Department of Internal Medicine, University Hospital Alfredo Lanari, Buenos Aires, Argentina); Silvia Attorri (Hospital Luis Lagomaggiore, Mendoza, Argentina); Enrique Barimboim (Hospital Central de Mendoza, Argentina); Juan Pablo Caeiro, María I Garzón (Hospital Privado Universitario, Córdoba, Argentina); Victor Hugo Cambursano (V H Dr Cazaux A Servicio de Neumologia, Hospital Rawson, Córdoba, Argentina); Adrian Ceccato (Hospital Nacional Prof Alejandro Posadas, Argentina); Julio Chertcoff , Florencia Lascar, Fernando Di Tulio (Critical Care Unit and Respiratory Medicine, Buenos Aires British Hospital, Buenos Aires, Argentina); Ariel Cordon Díaz (Hospital General Alvear, Ciudad, Mendoza, Argentina); Lautaro de Vedia (Respiratory Intensive Care Unit, Hospital Muñiz, Buenos Aires, Argentina); Maria Cristina Ganaha (Infectious Diseases Ward, Hospital Interzonal General de Agudos, Vicente Lopez y Planes from General Rodriguez, Buenos Aires, Argentina); Sandra Lambert (Hospital El Cruce - Alta Complejidad en Red, Argentina); Gustavo Lopardo, Hospital Bernardo Houssay, Vicente López, Argentina); Carlos M Luna (Pulmonary Medicine Division, Department of Medicine, Hospital de Clínicas, Universidad de Buenos Aires, Argentina); Alessio Gerardo Malberti (Hospital Nuestra Señora
}

del Carmen, Argentina); Nora Morcillo and Silvina Tartara (Hospital Zonal Especializado de Agudos y Crónicos Dr Antonio A Cetrangolo, Argentina); Claudia Pensotti (Infectious Diseases and Infection Control Department, Buenos Aires, Clinica Privada Monte Grande, Argentina); Betiana Pereyra (Hospital San Roque, Córdoba, Argentina); Pablo Gustavo Scapellato (Infectious Diseases Department, Hospital D F Santojanni, Argentina); Juan Pablo Stagnaro (HZGA Mi Pueblo, Florencio Varela, Argentina). Australia-Sonali Shah (Department of General Medicine, Austin Hospital, Heidelberg, Australia). Austria-Felix Lötsch, Florian Thalhammer (Division of Infectious Diseases and Tropical Medicine, Department of Medicine I, Medical University of Vienna, Austria). Belgium —Jean Louis Vincent (Department of Intensive Care, Erasme University Hospital, Université Libre de Bruxelles, Brussels, Belgium); Kurt Anseeuw (ZNA Campus Stuivenberg, Antwerp, Belgium); Camille A Francois (Anesthesia and critical care department, Erasme university hospital, Brussels, Belgium); Eva Van Braeckel (Department of Respiratory Medicine, Ghent University Hospital, Ghent, Belgium). Benin-Marcel Zannou Djimon, Jules Bashi, Dodo Roger (Centre Hospitalier Universitaire HKM of Cotonou, Benin). Brazil_-Simone Aranha Nouér (Federal University of Rio de Janeiro, Rio de Janeiro, Brazil). Bulgaria_-Peter Chipev, Milena Encheva (Clinic of Pulmonary Diseases, Military Medical Academy, Sofi a, Bulgaria); Darina Miteva (UMHAT "St. Marina", Varna, Bulgaria); Diana Petkova (University Hospital Varna, Bulgaria. Cameroon—Balkissou Adamou Dodo (Yaounde Jamot Hospital, Yaounde, Cameroon); Mbatchou Ngahane Bertrand Hugo (Douala General Hospital, Douala, Cameroon). China-Ning Shen (Respiratory Medicine, Peking University Third Hospital, Beijing, China); Jin-fu Xu, (Department of Respiratory Medicine, Shanghai Pulmonary Hospital, Tongji University, China). Colombia- Carlos Andres Bustamante Rico, Ricardo Buitrago (Clinica Shaio, Bogota, Colombia); Fernando Jose Pereira Paternina (Las Americas Clinic, Medellin, Colombia). Congo-Kayembe Ntumba Jean-Marie (Cliniques Universitaires de Kinshasa, DR Congo). Croatia-Vesna Vladic Carevic (Interne Medicine, Dubrovnik, Croatia); Marko Jakopovic (Medical School, University of Zagreb, Department for Respiratory Diseases Jordanovac, University Hospital Centre Zagreb, Zagreb, Croatia); Mateja Jankovic (University Hospital Center Zagreb, Department for Respiratory Diseases, Zagreb, Croatia); Zinka Matkovic (University Hospital Dubrava, Zagreb, Croatia); Ivan Mitrecic (Karlovac general hospital, Karlovac, Croatia). Denmark-Marie-Laure Bouchy Jacobsson (Emergency Department in North Zealand Hospital - Hillerød, Denmark); Anette Bro Christensen (Department of Anaethesiology, Viborg Region Hospital, Denmark); Uff e Christian HeitmannBødtger (Department of Pulmonology, Naestved Hospital, Denmark); Christian Niels Meyer (Department of Internal Medicine, Roskilde Hospital, Copenhagen University Hospital, Roskilde, Denmark); Andreas Vestergaard Jensen, Gertrud Baunbæk-knudsen, Pelle Trier Petersen and Stine Andersen (Department of Lung and Infectious Diseases, Nordsjællands HospitalHillerød, Denmark). Egypt-Ibrahim El-Said Abd El-Wahhab (Thoracic Medicine, Faculty of Medicine, Mansoura University, Egypt); Nesreen Elsayed Morsy (Pulmonary, Critical Care and Sleep Medicine, Faculty of Medicine, Mansoura University, Mansoura, Egypt); Hanaa Shafi ek (Chest diseases department, Faculty of Medicine, Alexandria University, Egypt); Eman Sobh (Chest Diseases Department, Al-Azhar University, Cairo, Egypt). France - Fabrice Bertrand (Critical care Unit, Robert Ballanger Hospital, Aulnay sous Bois, France); Christian Brun- Buisson (Univ Hospital Henri Mondor, 94000 Créteil, France); Etienne de Montmollin (Intensive care unit, Hôpital Delafontaine, Centre hospitalier de Saint-Denis, Saint-Denis, France); Muriel Fartoukh (Unité de réanimation médico-chirurgicale, Pôle Thorax Voies aériennes, Hôpital Tenon, Groupe Hospitalier Est Parisien, France); Jonathan Messika (Publique-Hôpital de Paris, Service de Réanimation Médicochirurgicale, Hôpital Louis Mourier, Colombes, France, and Université Paris Diderot, IAME, UMR 1137, Sorbonne Paris Cité, Paris, France); Pierre Tattevin (Infectious Diseases \& ICU, Pontchaillou University Hospital, Rennes, France). Germany - Michael Dreher (Department of Cardiology, Pneumology, Vascular Medicine and Intensive Care Medicine, University Hospital Aachen, Aachen, Germany); Martin Kolditz (Division of Pulmonology, Medical Department I, University Hospital Carl Gustav Carus, Technische Universität Dresden, Germany); Matthias Meisinger (Klinikum Niederlausitz GmbH, Klinik für Innere Medizin und Intensivmedizin, Senftenberg, Germany); Mathias W Pletz and Stefan Hagel (Center for Infectious Diseases and Infection Control, Jena University Hospital, Germany); Jan Rupp (Department of Molecular and Infectious Diseases, University of Lübeck, Lübeck, Germany); Tom 
Schaberg (Zentrum für Pneumologie, Agaplesion Diakonieklinikum Rotenburg, Germany); Marc Spielmanns (Internal Medicine Department, Pulmonary rehabilitation and Department of Health, School of Medicine, University Witten- Herdecke, St Remigius-Hospital, Leverkusen, Germany). Ghana-Beatrice Siaw-Lartey (Komfo-Anokye Teaching Hospital, Kumasi, Ghana). Greece-Katerina Dimakou (5th Rerpiratory Medicine Dpt, "SOTIRIA" Chest Hospital, Athens, Greece); Dimosthenis Papapetrou (Medical Group of Athens, Paleo Faliro Clinic, Athens, Greece); Evdoxia Tsigou and Dimitrios Ampazis, Agioi Anargiroi Hospital, Kifi ssia, Athens, Greece). India—Mohit Bhatia (S S Hospital IMS BHU Varanasi, India); Raja Dhar (Fortis Hospitals, Kolkata, India); George D'Souza (Department of Pulmonary Medicine, St John's Medical College Hospital, Bangalore, India); Rajiv Garg (Department of Respiratory Medicine, King George's Medical University UP, Lucknow, India); Parvaiz A Koul (Department of Internal \& Pulmonary Medicine, SheriKashmir Institute of Medical Sciences, Srinagar, India); P A Mahesh and B S Jayaraj (Department of Pulmonary Medicine, JSS Medical College, JSS University, Mysore, India); Kiran Vishnu Narayan (Pulmonary Medicine, Government Medical College Kozhikode, Kerala, India); Hirennappa B Udnur and Shashi Bhaskara Krishnamurthy (Columbia Asia Hospital, Hebbal, Bengaluru, Karnataka, India). Iran-Keihan Golshani (Isfahan University of Medical Sciences, Iran). Ireland-Vera M Keatings (Letterkenny General Hospital, Co. Donegal, Ireland); Ignacio Martin-Loeches (Multidisciplinary Intensive Care Research Organization (MICRO), St James's University Hospital, Trinity Centre for Health Sciences Dublin, Ireland). Israel_Yasmin Maor (Infectious Disease Unit, Affi liated to Tel Aviv University, Wolfson Medical Center, Holon, Israel); Jacob Strahilevitz (Department of Clinical Microbiology \& Infectious Diseases, Hadassah-Hebrew University, Jerusalem, Israel). Italy—Salvatore Battaglia (University of Palermo, Pneumologia DiBiMIS, Palermo, Italy); Maria Carrabba (Internal Medicine Department, Fondazione IRCCS Ca' Granda Ospedale Maggiore Policlinico, Milano, Italy); Piero Ceriana (Pulmonary rehabilitation, IRCCS Fondazione Maugeri, Pavia Italy); Marco Confalonieri (Department of Pulmunology, University Hospital, Trieste, Italy); Antonella d'Arminio Monforte (Department of Health Sciences, Clinic of Infectious Disease, San Paolo Hospital, University of Milan, Italy); Bruno Del Prato (Interventional Pneumology, Hospital Antonio Cardarelli, Naples, Italy); Marino De Rosa (UOC Pneumologia San Filippo Neri ASL RM E, Rome, Italy); Riccardo Fantini (Respiratory Diseases Clinic, Policlinico di Modena, Modena, Italy); Giuseppe Fiorentino (UOC Fisiopatologia e Riabilitazione Respiratoria AO Ospedali dei Colli PO, Monaldi, Italy); Maria Antonia Gammino (Pulmonary Medicine Unit, San Martino Hospital, ASL 5 Oristano, Sardegna, Italy); Francesco Menzella (Department of CardiacThoracic-Vascular and Intensive Care Medicine, Pneumology Unit, IRCCSArcispedale Santa Maria Nuova, Reggio Emilia, Italy); Giuseppe Milani (Azienda Ospedaliera Sant Anna di Como, Presidio Ospedale S Anna Nuovo, Unità Operativa di Pneumologia, Como, Italy); Stefano Nava (Alma Mater University of Bologna, DIMES, Respiratory and Critical Care Unit Sant'Orsola Malpighi Hospital, Italy); Gerardo Palmiero (Respiratory Unity, Versilia Hospital, Azienda USL 12 Viareggio, Lido di Camaiore, Lucca, Italy); Roberta Petrino and Barbra Gabrielli (Emergency Medicine Unit, S. Andrea Hospital, Vercelli, Italy); Paolo Rossi (Internal Medicine Department, Azienda Ospedaliero-Universitaria S. Maria della Misericordia, Udine, Italy); Claudio Sorino (Pulmonology Unit, AO Sant'Anna di Como, Italy); Gundi Steinhilber (Spedali Civili Brescia, UO Pneumologia e Fisiopatologia Respiratoria, Brescia, Italy); Alessandro Zanforlin (ULSS 18 Rovigo, Ospedale San Luca, Trecenta, Italy). Japan—Kiyoyasu Kurahashi (Yokohama City University Medical Center, Japan). Lebanon-Zeina Aoun Bacha (Medicine school, St Joseph University, Beyrouth, Lebanon). Mexico-Daniel Barajas Ugalde (National Institute of Respiratory Diseases, Mexico); Omar Ceballos Zuñiga (Hospital General de Mexicali, Mexicali, Baja California, Mexico); José F Villegas (Hospital Universitario Monterrey, México). Montenegro-Milic Medenica, Hospital for Lung Diseases_Brezovik, Niksic, Montenegro). Netherlands_E M W van de Garde (Dept. Clinical Pharmacy, St Antonius Hospital, Utrecht/ Nieuwegein, Netherlands). Nepal-Deebya Raj Mihsra (Internal Medicine, BP Koirala Institute of Health Sciences, Nepal); Poojan Shrestha, Oxford University Clinical Research Unit, Patan Hospital, Nepal). New Zealand-Elliott Ridgeon (Medical Research Institute of New Zealand).
Nigeria_Babatunde Ishola Awokola (Department of Family Medicine \& Primary Care, Lily Hospitals Limited, Warri, Nigeria); Ogonna N O Nwankwo (University of Calabar Teaching Hospital, Calabar, Nigeria); Adefuye Bolanle Olufunlola (Olabisi Onabanjo University teaching hospital, Sagamu, Ogun State, Nigeria); Segaolu Olumide (Department of Medicine, Pulmonary Unit, University College Hospital, Ibadan, Nigeria); Kingsley N Ukwaja (Department of Medicine, Federal Teaching Hospital Abakaliki, Ebonyi State, Nigeria). Pakistan-Muhammad Irfan (Section of Pulmonary and Critical Care Medicine, Department of Medicine, Aga Khan University, Karachi, Pakistan). Poland-Lukasz Minarowski (Department of Lung Diseaes and Tuberculosis, Medical University of Bialystok, Poland); Skoczyński Szymon (Department of Pneumology, School of Medicine in Katowice, Medical University of Silesia, Katowice, Institute of Occupational Medicine and Environmental Health, Sosnowiec, Poland). Portugal-Felipe Froes (Hospital Pulido Valente - CHLN, Lisboa, Portugal); Pedro Leuschner (Centro Hospitalar do Porto, Porto, Portugal); Mariana Meireles, Cláudia Ferrão, Pedro Leuschner and João Neves (Serviço de Medicina, Centro Hospitalar do Porto, Largo, Abel Salazar, Porto, Portugal); Sofi a B Ravara (Faculty of Health Sciences, University of Beira Interior); Cova da Beira Hospital Center, Covilhã, Portugal). Moldova-Victoria Brocovschii (Department of Pneumology \& Allergology, State University of Medicine and Pharmacy "Nicolae Testemitanu", Moldova); Chesov Ion (Clinic of Anesthesia and Intensive Care "Valeriu Ghrerg", Institute of Emergency Medicine, State University of Medicine and Pharmacy "Nicolae Testemitanu", Chisinau, Moldova); Doina Rusu (SMFU "N Testemitanu", Chisinau, Moldova); Cristina Toma (Department of Pneumology \& Allergology, State University of Medicine and Pharmacy "Nicolae Testemitanu", Chisinau, Moldova).

Romania-Daniela Chirita (Hospital Sfantul Stefan, Bucharest, Romania). Russia_Alexei Birkun (Department of Anesthesiology, Critical Care and Emergency Medicine, Medical Academy named after S I Georgievsky, Russia); Anna Kaluzhenina (Volgograd State Medical University, Russia). Saudi Arabia_Abdullah Almotairi (King Fahad medical City (KFMC), Riyadh, Saudi Arabia); Zakeya Abdulbaqi Ali Bukhary (College of Medicine, Taibah University, Medina, Saudi Arabia); Jameela Edathodu (Al Faisal University, King Faisal Specialist Hospital, Riyadh, Saudi Arabia); Amal Fathy (Pulmonary and respiratory critical care Medicine, Mansoura University Egypt, Affi liate at Taibah University, Saudi Arabia); Abdullah Mushira Abdulaziz Enani and Nazik Eltayeb Mohamed (Infectious Diseases Section, Medical Specialties Department, King Fahad Medical City, Riyadh, Saudi Arabia); Jawed Ulhadi Memon (Pulmonology Division, Department of Internal Medicine, King Fahad Hospital, Hofuf, Al Ahasa, 31982, Saudi Arabia). Serbia-Nada Bogdanović (Pulmonary department of KHC Dr Dragiša Mišović, Belgrade, Serbia); Branislava Milenkovic (Clinic for Pulmonary Diseases, Clinical Centre of Serbia, Faculty of Medicine, University of Belgrade, Belgrade, Serbia); Dragica Pesut (University of Belgrade School of Medicine, Teaching Hospital of Pulmonology, Clinical Centre of Serbia, Belgrade, Serbia). Spain—Luis Borderìas, Respiratoy and Sleep Unit, Hospital San Jorge, Huesca, Spain); Noel Manuel Bordon Garcia (Barcelona Policlínic and Moises Broggi Hospital at sant Joan Despí, Spain); Hugo Cabello Alarcón, Sant Hospital Seu de Urgell, Catalonia, Spain); Catia Cilloniz and Antoni Torres (Department of Pneumology, Institut Clinic del Tórax, Hospital Clinic of Barcelona Institut d'Investigacions Biomèdiques August Pi i Sunyer (IDIBAPS), University of Barcelona, Ciber de Enfermedades Respiratorias (CIBERES), Spain); Vicens Diaz-Brito and Xavier Casas (Infectious diseases Unit and Pneumology Service, Parc Sanitari Sant Joan de Deu, Sant Boi, Barcelona, Spain); Alicia Encabo González (Hospital Complex of Pontevedra, Spain); Maria Luisa FernándezAlmira (Medicina Interna, Hospital Universitario Central de Asturias, Spain); Miguel Gallego (Department of Respiratory Medicine, Hospital de Sabadell, Institut Universitari Parc Taulí-UAB, Sabadell, Spain. CIBER de Enfermedades Respiratorias, CIBERES, Bunyola, Spain); Inmaculada Gaspar-Garcla (Department of Respiratory Medicine, Hospital Costa del Sol, Marbella, Málaga, Spain); Juan González del Castillo (Emergency Department, Hospital Universitario Clínico San Carlos, Madrid, Spain); Patricia Javaloyes Victoria (Hospital General Universitario de Alicante, Alicante, Spain); Elena Laserna Martínez (Hospital Mollet, Barcelona, Spain); Rosa Malo de Molina (University Hospital Puerta de Hierro Majadahonda, Madrid); Pedro J Marcos (Servicio de Neumología, 
Complejo Hospitalario Universitario de A Coruña CHUAC, INIBIC, Sergas, Universidade de A Coruña, Spain); Rosario Menéndez (Pneumology Service, Universitary and Polytechnic Hospital La Fe, Valencia, Spain); Ana Pando-Sandoval (Hospital Universitario Central de Asturias. Area de Gestion Clinica de Pulmon. Servicio de Neumologia, Oviedo, Spain); Cristina Prat Aymerich, Alicia Lacoma del la Torre, and Ignasi García-Olivé (Microbiology Department and Pneumology Department, Hospital Universitari Germans Trias i Pujol, Institut d'Investigació Germans Trias i Pujol, Badalona, Spain; Universitat Autònoma de Barcelona; CIBER Enfermedades Respiratorias, Instituto de Salud Carlos III, Spain); Jordi Rello and Silvia Moyano (Critical Care Department, Hospital Vall d`Hebron, Barcelona, Spain); Francisco Sanz (Servicio de Neumología, Consorci Hospital General Universitari de Valencia, Valencia, Spain); Oriol Sibila and Ana Rodrigo-Troyano (Servei de Pneumologia, Hospital de la Santa Creu i Sant Pau, IIB-Sant Pau, Barcelona, Spain); Jordi Solé-Violán (Hospital Universitario de Gran Canaria Dr Negrín, Las Palmas de Gran Canaria, Spain); Ane Uranga (Pulmology Department, Hospital of Galdakao-Usansolo, Spain); Job FM van Boven (Hospital Universitari Son Espases, Palma de Mallorca, Spain); Ester Vendrell Torra and Jordi Almirall Pujol (Intensive Care Medicine, Hospital de Mataró, Spain). South Africa-Charles Feldman (Division of Pulmonology, Department of Internal Medicine, Charlotte Maxeke Johannesburg Academic Hospital, Faculty of Health Sciences, University of the Witwatersrand,

Johannesburg, South Africa). South Korea-Ho Kee Yum (Inje Univ. Seoul Paik Hospital, South Korea). Togo_Arnauld Attannon Fiogbe (Pulmonology and Infectious Diseases Service/University hospital of Sylvanus Olympio, Lomé, Togo). Tunisia—Ferdaous Yangui (Department of Pneumology, Hospital of Internal Forces Security (I.F.S), Marsa, Tunis, Tunisia). Turkey_-Semra Bilaceroglu (Izmir Dr Suat Seren Training and Research Hospital for Thoracic Medicine and Surgery, Izmir, Turkey); Levent Dalar (Pulmonary Medicine, Istanbul Bilim University, Istanbul, Turkey); Ufuk Yilmaz (Suat Seren Chest Disease and Surgery Training and Research Hospital, Izmir, Turkey). Ukraine-Artemii Bogomolov (Vinnitsa National Pirogov Memorial Medical University, Vinnitsa regional antituberculosis hospital, Vinnitsa, Ukraine). United Arab Emirates-Naheed Elahi (Dubai Hospital, UAE.); UK—Devesh J Dhasmana (Victoria Hospital, Kirkcaldy, NHS Fife, UK); Rhiannon Ions, Julie Skeemer, and Gerrit Woltmann (University Hospitals of Leicester NHS Trust and University of Leicester, Leicester, UK); Carole Hancock (Royal Respiratory Research Team, Royal Liverpool University Hospital, Liverpool, UK); Adam T Hill (Royal Infi rmary and University of Edinburgh, UK); Banu Rudran (The Royal London Hospital, Barts Health Trust, London, UK); Silvia RuizBuitrago and Marion Campbell (Hairmyres Hospital, Eaglesham Road, East Kilbride, UK); Paul Whitaker (Department of Respiratory Medicine, St James's Hospital, Leeds, UK). USA-Karen S Allen (University of Oklahoma Health Sciences Center, OK, USA); Veronica Brito (Texas A\&M Health Science Center, Division of Pulmonary, Critical Care and Sleep Medicine Baylor Scott \& White Health, TX, USA); Jessica Dietz (Fargo VA Health Care System, Fargo, ND, USA); Claire E Dysart and Susan M Kellie (Clement J Zablocki VA Medical Center, Milwaukee, WI, USA, Division of Infectious Diseases, University of New Mexico School of Medicine, Raymond G Murphy VA Medical Center, Albuquerque, NM, USA); Ricardo A Franco-Sadud and Garnet Meier (Division of Hospital Medicine, Cook County Hospital, Chicago, MI, USA); Mina Gaga (7th Resp Med Dept and Asthma Center, Athens Chest Hospital, USA); Thomas L Holland and Stephen P Bergin (Department of Medicine, Duke University Medical Center and School of Medicine, Duke Clinical Research Institute, NC, USA); Fayez Kheir (Department Pulmonary Diseases, Critical Care \& Environmental Medicine, Tulane University Health Sciences Center, New Orleans, LA, USA); Mark Landmeier (Division of Pulmonary and Critical Care Medicine, Northwestern Memorial Hospital, Chicago, IL, USA); Manuel Lois (John Peter Smith Hospital, Fort Worth, TX, USA); Girish B Nair (Interstitial Lung Disease Program and Pulmonary Rehabilitation, SUNY Stony Brook Winthrop University Hospital, Mineola, NY, USA); Hemali Patel (Department of Medicine, Division of General Internal Medicine, Hospital Medicine Group, University of Colorado, USA); Katherine Reyes (Henry Ford Hospital, Detroit, IL, USA); William Rodriguez-Cintron (Pulmonary/Critical Care Medicine VA Caribbean Healthcare System, USA); Shigeki Saito (Tulane University, New Orleans,
USA); Nilam J Soni, Julio Noda, Cecilia I Hinojosa, Stephanie M Levine, Luis F Angel, and Antonio Anzueto (Divisions of Hospital Medicine \& Pulmonary/Critical Care Medicine, South Texas Veterans Health Care System, University of Texas Health Science Center San Antonio, San Antonio, TX, USA); K Scott Whitlow, John Hipskind, and Kunal Sukhija (Kaweah Delta Health Care District, Department of Emergency Medicine, Visalia, CA, USA); Richard G. Wunderink and Ray D Shah (Northwestern University Feinberg School of Medicine, Chicago, IL, USA). Zambia_Kondwelani John Mateyo (Department of Internal Medicine, University Teaching Hospital, Lusaka, Zambia).

\section{Ethics approval and consent to partecipate}

The coordinating center (University of Texas Health at San Antonio -UT Health- Texas, USA) received project approval by the Institutional Review Board (IRB\# HSC20150184E). All participating centers followed their local law and regulations.

\section{Avaibility of data and materials}

The database used for the study is not public but it is available from the corresponding author on reasonable request.

\section{Funding}

The authors did not receive any funding for this study.

\section{Authors' contributions}

AG, SA and GS analyzed the dataset. AG, SA, GS, MDP, DR and ST interpreted the patient data. LFR took charge of Figs. EV, JN, FM, FB and MIR along with all the auothors read and approved the final manuscript.

\section{Consent for publication}

Not applicable.

\section{Competing interests}

The authors declare that they have no competing interests for this study.

\section{Publisher's Note}

Springer Nature remains neutral with regard to jurisdictional claims in published maps and institutional affiliations.

\section{Author details}

'Department of Pathophysiology and Transplantation, University of Milan, Internal Medicine Department, Respiratory unit and Adult Cystic Fibrosis Center, Fondazione IRCCS Ca' Granda Ospedale Maggiore Policlinico, Via Francesco Sforza 35, 20122 Milan, Italy. ${ }^{2}$ Clinical Epidemiology and Medical Statistics Unit, Department of Clinical and Experimental Medicine, University of Sassari, Sassari, Italy. ${ }^{3}$ Department of Biomedical and Clinical Sciences (DIBIC), University of Milan, Section of Respiratory Diseases, Ospedale L. Sacco, ASST Fatebenefratelli-Sacco, Milan, Italy. ${ }^{4}$ Respiratory Unit, San Paolo Hospital, Department of Medical Sciences, University of Milan, Milan, Italy. ${ }^{5}$ Division of Pulmonary Diseases and Critical Care Medicine, The University of Texas Health Science Center at San Antonio, San Antonio, TX, USA. Intensive Care Unit, Hospital de Matarò, Consorci Sanitari del Maresme, Carretera de Cirera s/n, 08304 Matarò, Barcelona, Spain. ${ }^{7}$ Internal Medicine Department, Centro Hospitalar do Porto, Porto, Portugal. ${ }^{8}$ Department of Medical Specialties, Pneumology Unit, IRCCS Arcispedale Santa Maria Nuova, Azienda USL Reggio Emilia, Italy.

Received: 28 May 2018 Accepted: 28 November 2018 Published online: 18 December 2018

\section{References}

1. Mandell LA, Wunderink RG, Anzueto A, et al. Infectious Diseases Society of America; American Thoracic Society. Infectious Diseases Society of America/ American Thoracic Society consensus guidelines on the management of community-acquired pneumonia in adults. Clin Infect Dis. 2007;44(suppl 2): S27-72.

2. Ramirez JA, Wiemken TL, Peyrani P, Arnold FW, Kelley R, Mattingly WA, Nakamatsu R, Pena S, Guinn BE, Furmanek SP, Persaud AK, Raghuram A, Fernandez F, Beavin L, Bosson R, Fernandez-Botran R, Cavallazzi R, Bordon J, Valdivieso C, Schulte J, Carrico RM. University of Louisville Pneumonia Study 
Group. Adults hospitalized with pneumonia in the United States: incidence, epidemiology, and mortality. Clin Infect Dis. 2017 Nov 13;65(11):1806-12.

3. Eliakim-Raz N, Robenshtok E, Shefet D, Gafter-Gvili A, Vidal L, Paul M, Leibovici L. Empiric antibiotic coverage of atypical pathogens for community-acquired pneumonia in hospitalized adults. Cochrane Database of Systematic Reviews 2012, Issue 9. Art. No.: CD004418.

4. Arnold FW, Summersgill JT, Ramirez JA. Role of atypical pathogens in the etiology of community-acquired pneumonia. Semin Respir Crit Care Med. 2016 Dec;37(6):819-28 Epub 2016 Dec 13.

5. Tao LL, Hu BJ, He LX, Wei L, Xie HM, Wang BQ, Li HY, Chen XH, Zhou CM, Deng WW. Etiology and antimicrobial resistance of community-acquired pneumonia in adult patients in China. Chin Med J. 2012 Sep;125(17):2967-72.

6. Arnold FW, Summersgill JT, Lajoie AS, Peyrani P, Marrie TJ, Rossi P, Blasi F, Fernandez P, File TM Jr, Rello J, Menendez R, Marzoratti L, Luna CM, Ramirez JA. Community-acquired pneumonia organization (CAPO) investigators. A worldwide perspective of atypical pathogens in community-acquired pneumonia. Am J Respir Crit Care Med. 2007 May 15;175(10):1086-93.

7. Woodhead M, Blasi F, Ewig S, Garau J, Huchon G, leven M, Ortqvist A, Schaberg T, Torres A, van der Heijden G, Read R, Verheij TJ. Joint taskforce of the European Respiratory Society and European Society for Clinical Microbiology and Infectious Diseases. Guidelines for the management of adult lower respiratory tract infections-full version. Clin Microbiol Infect. 2011 Nov; 17(Suppl 6):E1-59. https://doi.org/10.1111/j.1469-0691.2011.03672.x.

8. Aliberti S, Reyes LF, Faverio P, Sotgiu G, Dore S, Rodriguez AH, Soni NJ, Restrepo MI, investigators GLIMP. Global initiative for meticillin-resistant Staphylococcus aureus pneumonia (GLIMP): an international, observational cohort study. Lancet Infect Dis. 2016 Dec;16(12):1364-76. https://doi.org/10. 1016/S1473-3099(16)30267-5.

9. Martinez MA, Ruiz M, Zunino E, Luchsinger V, Avendano LF. Detection of mycoplasma pneumoniae in adult community-acquired pneumonia by PCR and serology. J Med Microbiol. 2008;57(Pt 12):1491-5.

10. von BH, Welte T, Marre R, Suttorp N, Luck C, Ewig S. Mycoplasma pneumoniae pneumonia revisited within the German competence network for community-acquired pneumonia (CAPNETZ). BMC Infect Dis. 2009;9:62.

11. Thurman KA, Walter ND, Schwartz SB, et al. Comparison of laboratory diagnostic procedures for detection of mycoplasma pneumoniae in community outbreaks. Clin Infect Dis. 2009;48:1244-9.

12. Welte T, Suttorp N, Marre R. CAPNETZ: community-acquired pneumonia competence network. Infection. 2004;32:234-8.

13. Raeven VM, Spoorenberg SM, Boersma WG, van de Garde EM, Cannegieter SC, Voorn GP, Bos WJ, van Steenbergen JE, Alkmaar study group, Ovidius study group. Atypical aetiology in patients hospitalised with community-acquired pneumonia is associated with age, gender and season; a data-analysis on four Dutch cohorts. BMC Infect Dis. 2016 Jun 17;16:299. https://doi.org/10.1186/ s12879-016-1641-9.

14. Lim WS, Baudouin SV, George RC, et al. BTS guidelines for the management of community acquired pneumonia in adults: update 2009. Thorax 2009; 64: Suppl. 3, iii1-iiis5.

15. Yanagihara K, Kohno S, Matsusima T. Japanese guidelines for the management of community-acquired pneumonia. Int J Antimicrob Agents. 2001;18(Suppl. 1): S45-8.

16. Working Group of the South African Thoracic Society. Management of community-acquired pneumonia in adults. S Afr Med J. 2007;97:1296-306.

17. Singanayagam A, Aliberti S, Cillóniz C, et al. Evaluation of severity scoreguided approaches to macrolide use in community-acquired pneumonia. Eur Respir J. 2017;50:1602306.

18. Viasus D, Di Yacovo S, Garcia-Vidal C, Verdaguer R, Manresa F, Dorca J, Gudiol F, Carratalà J. Community- acquired legionella pneumophila pneumonia:a single- center experience with 214 hospitalized sporadic cases over 15 years. Medicine. 2012;92:51-60.

19. Vergis EN, Indorf A, File TM Jr, Phillips J, Bates J, Tan J, Sarosi GA, Grayston JT, Summersgill J, Yu VL. Azithromycin vs cefuroxime plus erythromycin for empirical treatment of community-acquired pneumonia in hospitalized patients: a prospective, randomized, multicenter trial. Arch Intern Med. 2000 May 8;160(9):1294-300.

20. Luchsinger V, Ruiz M, Zunino E, Martínez MA, Machado C, Piedra PA, Fasce $R$, Ulloa MT, Fink MC, Lara P, Gebauer M, Chávez F, Avendaño LF. Community- acquired pneumonia in Chile: the clinical relevance in the detection of viruses and atypical bacteria. Thorax. 2013;68:1000-6.

21. Spoorenberg SM, Bos WJ, Heijligenberg R, Voorn PG, Grutters JC, Rijkers GT, van de Garde EM. Microbial aetiology, outcomes, and costs of hospitalisation for community-acquired pneumonia; an observational analysis. BMC Infect Dis. 2014;14:335-43.

22. Shibli F, Chazan B, Nitzan O, Flatau E, Edelstein H, Blondheim O, Raz R, Colodner R. Etiology of community-acquired pneumonia in hospitalized patients in northern Israel. Isr Med Assoc J. 2010;12:477-82.

23. Chen K, Jia R, Li L, Yang C, Shi Y. The aetiology of community associated pneumonia in children in Nanjing, China and aetiological patternsassociated with age and season. BMC Public Health. 2015;15:113-8.

24. Arancibia F, Cortes CP, Valdés M, Cerda J, Hernández A, Soto L, Torres A. Importance of legionella pneumophila in the etiology of severe community-acquired pneumonia in Santiago. Chile Chest. 2014;145:290-6.

25. Templeton KE, Scheltinga SA, van den Eeden WC, Graffelman AW, van den Broek PJ, Claas EC. Improved diagnosis of the etiology of communityacquired pneumonia with real-time polymerase chain reaction. Clin Infect Dis. 2005:41:345-51.

\section{Ready to submit your research? Choose BMC and benefit from:}

- fast, convenient online submission

- thorough peer review by experienced researchers in your field

- rapid publication on acceptance

- support for research data, including large and complex data types

- gold Open Access which fosters wider collaboration and increased citations

- maximum visibility for your research: over $100 \mathrm{M}$ website views per year

At BMC, research is always in progress.

Learn more biomedcentral.com/submissions 